\title{
A Fabricação Robótica no Ensino da Arquitetura: Uma Experiência Sobre o Projecto e Construção de Estruturas em Tijolo
}

\author{
- José Pedro Sousa \\ Faculdade de Arquitectura da Universidade do \\ Porto + DFL/CEAU, Portugal \\ jsousa@arq.up.pt \\ - João Pedro Xavier \\ Faculdade de Arquitectura da Universidade do \\ Porto + DFL/CEAU, Portugal \\ jxavier@arq.up.pt
}

Robotic Fabrication in Architectural Education: An Experience on the Design and Construction of Brick Structures

\begin{abstract}
In the last decade, architectural researchers have demonstrated the potential of using robots to design and construct in novel ways. However, the integration of such practices in architectural education has been difficult and the examples are rare. By analyzing this context, this paper describes a teaching experience at FAUP where robotic technologies were introduced to the Master students for the first time. The assignment consisted in the production of a brick structure and ended up with the construction of a 1:1 scale installation. With this experience, this paper wants to contribute for the dissemination of robotic technologies in architectural curriculums.
\end{abstract}

Keywords: Architectural Education, Digital Fabrication, Robotics, 3D Printing, Brick Construction

\section{Introdução}

Hoje em dia, a fabricação digital éuma área crucial na produção arquitetónica contemporânea, pois viabiliza a materialização de soluções geométricas (ex: formas, estruturas, componentes construtivos...) que de outra forma dificilmente poderiam ser executadas (Kolarevic 2001). Entre as várias tecnologias existentes, a exploração de robots industriais tem assumido um papel cada vez mais relevante nos últimos anos, desde que Gramazio \& Kohler (2008) apresentaram os seus primeiros trabalhos recorrendo ao uso de braços robóticos em 2005. Nas suas construções pioneiras, os Professores da ETH Zurich mostraram como esta tecnologia, originalmente desenvolvida para outras indústrias baseadas em procedimentos repetitivos com forte potencial de automação (ex: automóvel), poderia ser usada pelos arquitetos para automatizar uma produção nãostandard e personalizada. A combinação dos seus 6 (ou mais) eixos de movimentos com a possibilidade de adaptação na sua flange de um sem número de ferramentas (ex: fresadora, garras, extrusores, fio quente...), faz com que o braço robótico seja a tecnologia de fabricação mais avançada, flexível e versátil da actualidade.

A evolução da utilização de robots na arquitetura tem vindo a crescer exponencialmente ao longo dos últimos dez anos, tendo, inclusivamente, originado a criação de uma organização específica denominada como Association for Robots in Architecture (www.robotsinarchitecture.org).
Em síntese, pode-se afirmar que esta evolução tem sido conduzida seguindo dois caminhos paralelos. Por um lado, verifica-se uma expansão da utilização de robots no campo da investigação em arquitetura, explorando materiais e sistemas construtivos inovadores. Os pavilhões de Verão projectados pelo ICD com o ITKE da University of Stuttgart desde 2010, constituem exemplos da exploração de novas formas de pensar e executar a materialidade em arquitetura (Menges 2012). Por outro lado, o conhecimento disciplinar crescente desta tecnologia, e a inspiração daí resultante, tem conduzido à sua progressiva transferência para a prática, centrando a sua aplicação sobretudo na pré-fabricação de componentes construtivos.

\section{Integração da Tecnologia de Fabricação Robótica no Ensino da Arquitetura}

Apesar das evidências do potencial criativo e material da tecnologia robótica demonstradas pela prática e investigação em arquitetura (Bechthold \& Kind 2012), são muito poucos os exemplos de integração no ensino ao nível da graduação. A justificar este facto, podem-se destacar as seguintes razões:

- Segurança (utilização);

- Complexidade (programação);

- Resistência (disciplinar).

Sobre a primeira, a utilização deste equipamento exige a adopção de procedimentos de segurança mais elevados que 
durante o manuseamento de outras máquinas de fabricação digital. A dimensão e a cinemática do robot definem um espaço de trabalho volumétrico de grande dimensão, bem diferente do espaço de trabalho plano e bem delimitado das máquinas CNC convencionais (Figura 1). Assim, durante a operação, os movimentos do robot podem provocar colisões e libertação de material de forma perigosa e inesperada (ex: a programação de trajectórias por PTP deixa ao robot a decisão sobre rotações e movimentos que deve efectuar para ir de um ponto a outro ponto). Por este motivo, a instalação do robot requer uma célula devidamente protegida e equipada com sistema de emergência para a interrupção do seu funcionamento.


Figura 1: O espaço volumétrico de trabalho do braço robótico do DFL que serve de base para a definição da sua célula de instalação (fonte: manual oficial da Kuka Robotics).

Paralelamente, sendo a máquina de fabricação digital mais flexível da actualidade, a utilização do braço robótico é um pouco mais complexa que outros equipamentos de fabricação digital (ex: corte a laser ou fresadora CNC). A sua programação envolve a definição de um maior número de parâmetros, necessários para controlar movimentos em vários eixos e coordená-los com a automação de comportamentos atribuídos às diferentes ferramentas (ex: garra, fresadora...) e aos dispositivos que se podem instalar (ex: sistemas de visão, sensores...). Esta condição de versatilidade de usos exige procedimentos de fabricação específicos para cada uma das aplicações. Juntando este facto ao anterior, é consideravelmente mais difícil de conceber a autonomia do aluno na utilização deste equipamento em comparação com outras máquinas de fabricação digital.

Finalmente, tal como aconteceu com outras máquinas precedentes, como o computador, a introdução do robot nas escolas de arquitetura não é pacífica. A sua assimilação disciplinar encontra uma forte resistência e desconfiança nas váriasáreas curriculares do ensino da arquitetura, desde a teoria e a história, à construção e ao projecto. Consequentemente, a sua integração é lenta e geralmente confinada a um pequeno grupo de docentes que têm um interesse específico na área da investigação das tecnologias digitais.

O conjunto das três razões descritas explica a dificuldade de integração da tecnologia robótica na formação ao nível da graduação do arquiteto.

\subsection{0 caso do Digital Fabrication Laboratory (DFL)}

Neste contexto, a introdução da robótica na instituição dos autores, a Faculdade de Arquitetura da Universidade do Porto (FAUP), não foi diferente. Surgiu por acção do recém criado grupo de investigação denominado Digital Fabrication Laboratory (DFL) e foi financiada através de um Projecto de Investigação apoiado pela Fundação para a Ciência e a Tecnologia. Iniciando este trabalho em 2013, o DFL adquiriu um robot de grandes dimensões (Kuka KR120 R2700 extra HA) e desenvolveu um amplo conjunto de trabalhos práticos à escala real, envolvendo diferentes materiais e técnicas de fabrico. Instalado num edifício geograficamente separado da FAUP, o DFL procura promover acções de disseminação das tecnologias robótica para além do domínio da sua investigação, como sejam, visitas dos alunos da FAUP e de outras escolas ao espaço do laboratório (Figura 2).

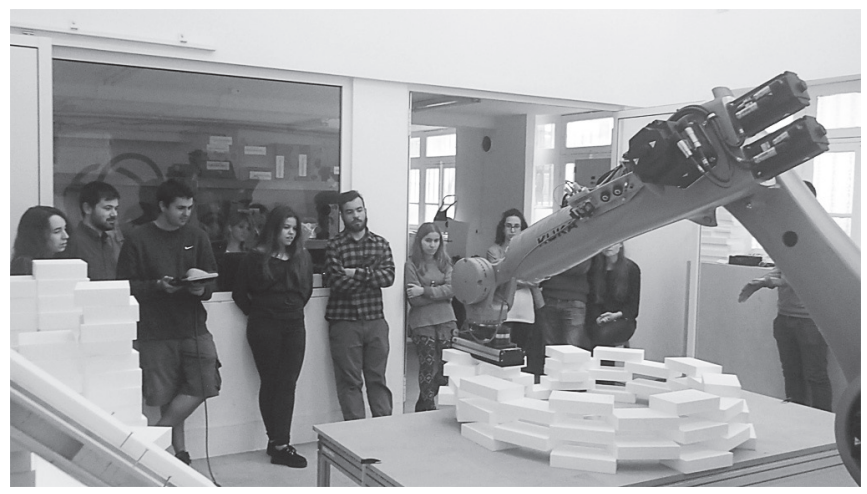

Figura 2: Visita ao DFL dos alunos de Geometria Construtiva da FAUP para introdução à utilização do braço robótico no âmbito da experiência descrita no presente artigo.

Neste contexto, foi na unidade curricular Geometria Construtiva do $3^{\circ}$ ano do curso de Mestrado Integrado em Arquitetura da FAUP que os autores realizaram, em Março e Abril de 2015, o primeiro exercício de introdução prática da tecnologia robótica aos alunos de arquitetura. O próximo capítulo descreve as várias fases de desenvolvimento desta experiência que procurou contribuir para uma melhor compreensão desta tecnologias no contexto de ensino, enquanto ferramentas de auxílio ao pensamento e materialização em arquitetura.

\section{Uma Experiência de Ensino}

\subsection{Enquadramento}

Como forma de introdução à fabricação robótica, propôsse o estudo do trabalho que Gramazio \& Kohler têm vindo a realizar na ETH de Zurique sobre estruturas em alvenaria de tijolo, destacando-se dois projectos. Por um lado, a instalação para a Bienal de Veneza de 2010 serviu de exemplo para explicar a problemática do exercício. Incidindo apenas sobre o posicionamento diferenciado de elementos standard -tijolos- no espaço, e não na fabricação da sua diferenciação 
geométrica, a tecnologia robótica permite a montagem de uma estrutura em alvenaria que dificilmente poderia ser executada com precisão e rapidez através de processos manuais. Por outro lado, a fachada do edifício da Gantenbein Winery de 2006, foi útil para demonstrar como estas tecnologias e processos podem passar à prática real da arquitectura com resultados inovadores.

\subsection{Descrição do Exercício}

Neste enquadramento, o exercício foi apresentado a uma turma de 42 estudantes, que se organizaram em grupos de 2 ou 3 alunos. O objectivo consistiu no projecto de uma estrutura em alvenaria com tijolos de EPS capaz de ser autoportante e de demonstrar uma aplicação pertinente da tecnologia. No final, os alunos teriam que escolher uma das soluções para ser fabricada roboticamente.

Este desafio teve a duração de 5 aulas e considerou os seguintes dados:

- dimensão do tijolo: $25 \times 25 \times 5 \mathrm{~cm}$

- $\quad$ quantidade disponível: 500

- $\quad$ processo de desenho/modelação: livre

- $\quad$ processo de fabricação: montagem robótica

\subsection{Desenvolvimento do trabalho}

Durante a fase de concepção, os alunos exploraram diferentes estratégias de desenho digital. Sendo a primeira unidade curricular da sua formação dedicada ao uso do computador, nem todos os alunos demonstram a mesma destreza e confiança a lidar com o meio digital. Enquanto que alguns modelaram de forma explícita o seu projecto em Rhinoceros, outros exploraram a modelação paramétrica com Grasshopper. Previsivelmente, esta segunda abordagem revelou-se mais flexível e poderosa para tratar de todo o processo de geração formal e da sua discretização em pequenos elementos -tijolos. Inicialmente, para permitir que a tecnologia e a materialidade pudessem contribuir na concepção das propostas, organizouse uma visita ao laboratório para que os alunos pudessem contactar com o robot e com o seu modo de funcionamento.

Durante a fase de concepção, os alunos exploraram diferentes estratégias de desenho digital. Sendo a primeira unidade curricular da sua formação dedicada ao uso do computador, nem todos os alunos demonstram a mesma destreza e confiança a lidar com o meio digital. Enquanto que alguns modelaram de forma explícita o seu projecto em Rhinoceros, outros exploraram a modelação paramétrica com Grasshopper. Previsivelmente, esta segunda abordagem revelou-se mais flexível e poderosa para tratar de todo o processo de geração formal e da sua discretização em pequenos elementos -tijolos. Inicialmente, para permitir que a tecnologia e a materialidade pudessem contribuir na concepção das propostas, organizou-se uma visita ao laboratório para que os alunos pudessem contactar com o robot e com o seu modo de funcionamento.

Igualmente, disponibilizaram-se um conjunto de tijolos em EPS na sala de aula para permitir ensaiar manualmente estratégias de assentamento (Figura 3).

Para uma melhor avaliação da geometria das soluções e da sua integridade estrutural, cada grupo teve que imprimir em 3D a sua proposta utilizando ABS e o equipamento do DFL, uma Makerbot Replicator 2x. Esta experiência foi bastante elucidativa do interesse geométrico das propostas e da qualidade dos modelos digitais. Permitiu detectar problemas como, por exemplo, zonas de intersecção entre tijolos, áreas de sobreposição insuficiente de tijolos, ou a ineficácia estrutural da sua geometria (Figura 4).

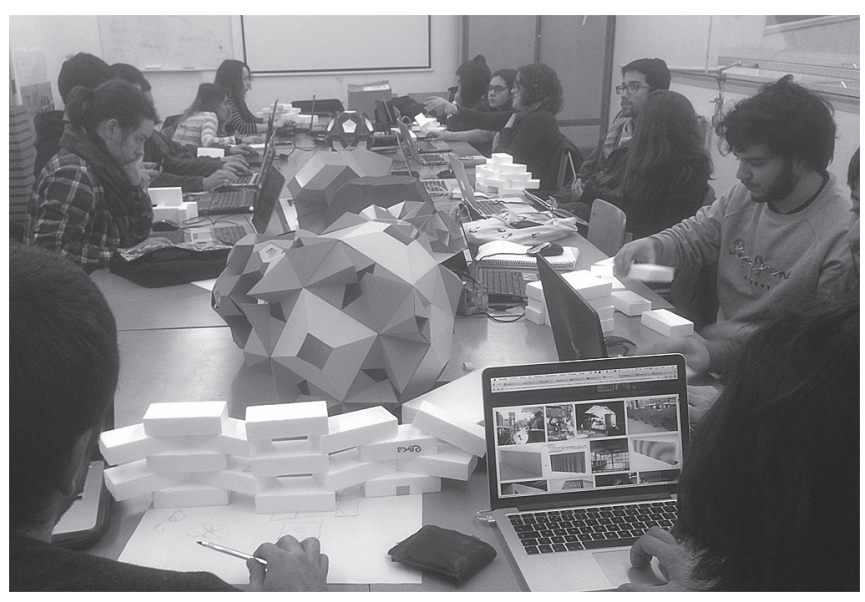

Figura 3: Trabalho na sala de aula, realizando experiências manuais com tijolos em EPS em simultâneo com o desenho e modelação digital.

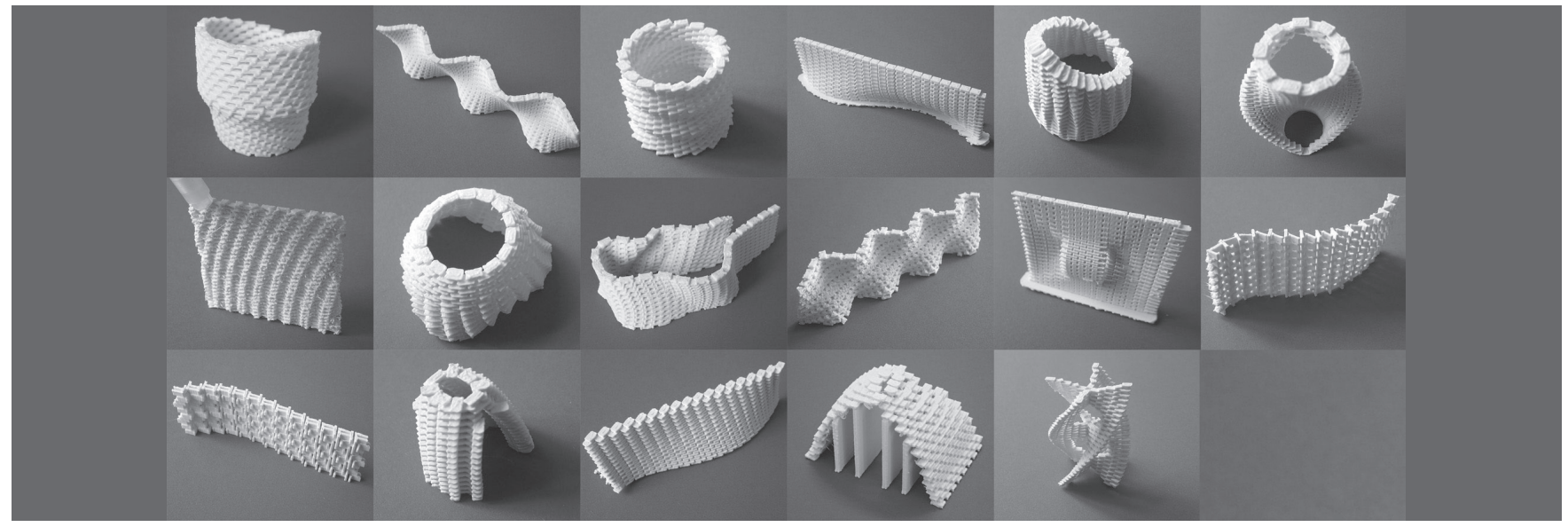

Figura 4: Modelos produzidos por impressão 3D em ABS das propostas desenvolvidas pelos alunos. 
Após 3 aulas de trabalho prático (incluindo a visita), cada grupo teve que apresentar à turma a sua proposta tendo em vista a selecção colectiva, por votação, da mais interessante e viável para ser fabricada roboticamente. Esta apresentação foi feita através de cartazes e das maquetas impressas em 3D (Figura 5).

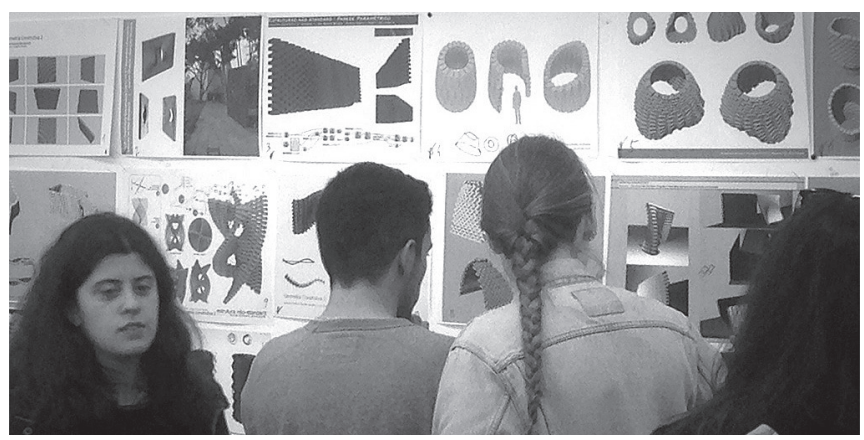

Figura 5: Momento de apresentação e discussão das propostas.

O conjunto destes elementos permitiu uma apreciação bastante completa da qualidade das propostas. A solução vencedora emergiu quase unanimemente. Os alunos Saule Grybenaite e Jorge Juan Pérez apresentaram uma forma bastante interessante, tanto do ponto de vista geométrico como estrutural, que acabou por convencer convenceu a opinião geral da turma (Figura 6).

Com os alunos presentes no laboratório do DFL, a programação do robot foi realizada em modo tutorial, recorrendo ao plugin KUKA/Prc para Grasshopper. Com esta ferramenta, definiram-se todos os passos de automação da montagem dos tijolos com recurso a garra por vácuo.

A alimentação dos tijolos para o robot foi feita a partir de um sistema de rampa construído para o feito. Uma vez
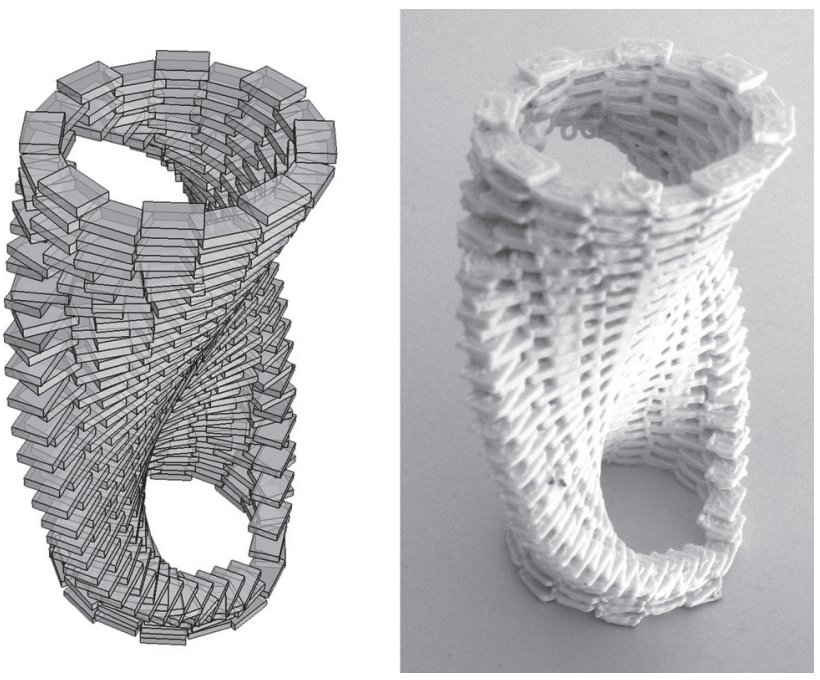

Figura 6: Modelo digital em Rhinoceros (à esquerda) e modelo impresso em 3D (direita) da proposta seleccionada para fabricação robótica.

que o processo de colagem não está ainda automatizado, efectuou-se uma paragem do robot após a colocação de cada nível de tijolos, para deposição manual de cola. Apesar do alcance do robot ser elevado, a dimensão da estrutura obrigou ao seu fabrico em 2 partes. No entanto, a geometria da solução apresentava uma simetria bilateral que induziu a estratégia natural para a sua divisão. $O$ processo de fabricação decorreu sem sobressaltos, e a estrutura final corresponde com muito rigor ao modelo digital (Figura 7).

Confirmando-se o seu comportamento autoportante, o impacto visual da estrutura construída à escala real foi do agrado de todos. A variação incremental da disposição dos tijolos no espaço beneficiou claramente do recurso à tecnologia robótica (Figura 8).
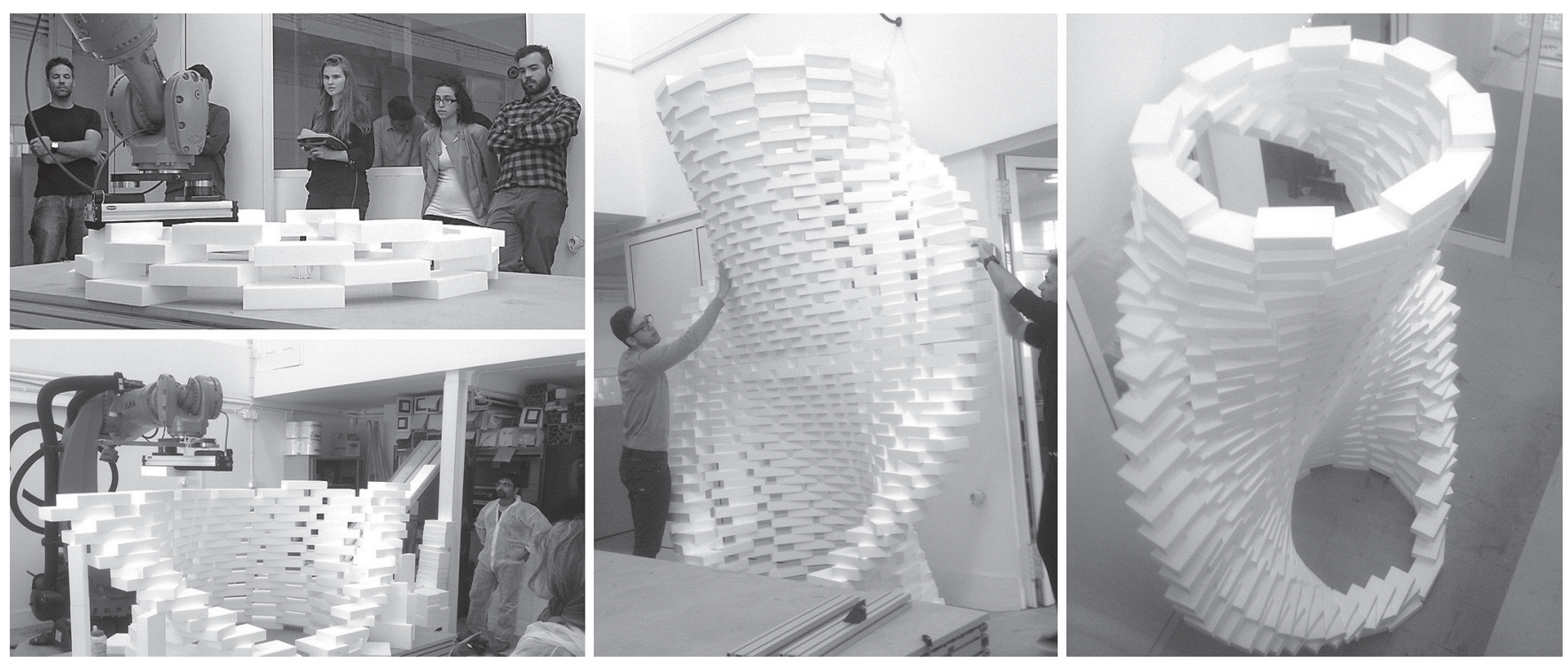

Figura 7: Fabricação robótica com intervenção assistida dos alunos no comando do robot (esquerda), montagem da estrutura através da sobreposição das duas partes fabricadas (centro), e vista superior da estrutura (direita)o desenho e modelação. 




Figura 8: Fotografia da instalação final na galeria do DFL.

\section{Conclusão}

Do ponto de vista material, os principais resultados desta experiência de ensino traduziram-se na produção de:

- 20 modelos digitais concebidos por diferentes técnicas digitais (ex: modelação, parametrização);

- 17 modelos físicos de impressão 3D;

- 1 estrutura fabricada roboticamente à escala 1:1, com 2,5 metros de altura.

Sob o ponto de vista da integração da fabricação robótica no ensino da arquitetura, esta primeira experiência comprovou a dificuldade em permitir uma autonomia doaluno no comando do equipamento, pelas razões apontadas no capítulo 2 . No entanto, a flexibilidade da aplicação utilizada para a programação do robot, o Kuka|Prc convida a pensar numa futura experiência de ensino com maior duração, na qual os alunos poderão garantir autónoma e virtualmente grande parte do processo de fabricação das suas soluções. A maioria dos alunos demonstrou curiosidade em saber mais sobre os modos de programação do robot tendo em vista poder integrar de um modo mais interactivo, as decisões de fabricação com as de projecto.

Metodologicamente, com este exercício, os estudantes puderam contactar com o desenvolvimento de um pequeno projecto, desde a sua concepção à sua materialização,
exclusivamenteatravésdemeiosdigitais.Ageometriadaestrutura construída foi clarificadora da pertinência da utilização das tecnologias de fabricação robótica nestes sistemas construtivos, face aos processos tradicionais de montagem manual. Seria muito difícil e impreciso compreender e montar manualmente uma estrutura com esta configuração. Paralelamente, o facto deste exercício propor a realização de um projecto a partir de uma possibilidade material e não de um programa, permitiu também recordar a influência que os materiais e os métodos construtivos podem ter na fundação tectónica do projecto. Alguns estudantes mostraram-se surpreendidos pela experiência, pois esta fonte de inspiração é muitas vezes relegada para segundo plano na prática de projecto, onde a solução material e construtiva tende a ser pensada numa etapa final do processo para viabilizar uma ideia formal de projecto. Seguindo as considerações de Brell-Çokcan \& Braumann (2013), com a evolução dos meios de programação robótica pode-se esperar que esta interactividade venha a ser mais fluída, estendendo a prática criativa e produtiva com auxílio dos meios digitais descrita por McCullough (1998).

Assim, a relação biunívoca entre o pensar e o fazer em arquitetura ficou patente neste exercício, que também proporcionou um contacto com a construçãoà escala 1:1, que não é muito frequente no ensino da arquitetura. Pela importância crescente desta tecnologia, espera-se que a realização deste tipo de experiências possa contribuir para a aproximação do seu uso à formação do futuro profissional de arquitetura.

\section{Acknowledgments}

Esta experiência foi desenvolvida no âmbito do Projecto de Investigação com a referência PTDC/ATP-AQI/5124/2012, financiado por Fundos FEDER através do Programa Operacional Factores de Competitividade - COMPETE e por Fundos Nacionais através da FCT - Fundação para a Ciência e a Tecnologia. No âmbito deste Projecto, a investigação sobre construção em tijolo conta com o apoio do parceiro industrial Cerâmica Vale da Gândara.

\section{References}

Bechthold,M.\& King, N.(2012). Rob|Arch2012-RoboticFabrication in Architecture, Art and Design, 118-130. Wien: Springer-Verlag. Brell-Çokcan, S. \& Braumann, J. (2013). Industrial Robots for Design Education: Robots as Open Interfaces beyond Fabrication. Communications in Computer and Information Science, Vol. 369, 109-117.

Gramazio F., \& Kohler, M. (2008). Digital Materiality. Basel: Lars Muller Publishers.

Kolarevic,B.(2001).DigitalFabrication:Manufacturing Architecture in the Information Age. Reinventing the Discourse, Proceedings of the ACADIA 2001 Conference, 268-277, Washington DC.4

McCullough, M. (1998). Abstracting Craft. The Practiced Digital Hand. MIT Press.

Menges, A. (Ed.) (2012). Material Computation: Higher Integration in Morphogenetic Architectural Design, Architectural Design, March 2012. 